\title{
ASSESSMENT OF BIOLOGICAL MARKERS LEVEL IN GINGIVAL CREVICULAR FLUIDS DURING EARLY ORTHODONTIC TREATMENT OF PERIODONTALLY AFFECTED TEETH
}

\author{
Fatma AbdelAziz*, Naglaa ElKilany** and Olfat Shaker ${ }^{* * *}$
}

\begin{abstract}
Background: the aim of the present study was to evaluate the levels of some biological markers; interleukin 1B (IL1B), interleukin 6 (IL6), interleukin 8 (IL8) and prostaglandin(PG) in the gingival crevicular fluids $(\mathrm{GCF})$ in patients with periodontal disease during early stage of orthodontic tooth movement.
\end{abstract}

Subjects and methods: The study included 9 subjects with age range 23-45 years, with periodontally affected teeth submitted to orthodontic treatment at the orthodontic department faculty of dentistry, Al-azhar University,Girls branch. Fixed appliances were inserted, nickel titanium light archwires were used for levelling and alignment. Gingival crevicular fluid (GCF) samples were collected from the healthy and periodontally affected teeth with paper points at base line, 3 days, $1,2,3,4$ weeks from starting of orthodontic treatment.

Results: during the test period, the levels of IL 6 in healthy teeth and periodontally affected teeth gradually increased after 3 days and 1 week, then gradually decreased after 2 and 3 weeks, Comparing healthy and periodontally affected teeth revealed a higher levels in periodontally affected teeth.. The mean value of IL 8 gradually increased after 3 days and 1 week, then gradually decreased after 2 and 3 weeks. the mean value of IL 1B was gradually increased after 3 days and 1 week, then gradually decreased after 2 and 3 week. PG showed gradual increase after 3 days and 1 week, then gradually decreased after 2,3 and 4weeks. Comparing healthy and periodontally affected teeth revealed a higher levels in periodontally affected teeth.

Conclusion: Our findings suggested that the orthodontic movement of periodontally compromised teeth result in significant changes in the levels of certain biological markers in the GCF as compared to healthy teeth.

* Assistant Professor at the Orthodontic Department, Faculty of Dental medicine for Girls, Al-Azhar University.

** Professor and Head of the Oral Radiology, Medicine, Periodontology and Diagnosis Department, Faculty of Dental Medicine for Girl's, Al-Azhar University.

**** Professor at Biochemistry Department, Faculty of Medicine, Cairo University. 


\section{INTRODUCTION}

The most common objectives of an orthodontic treatment are facial and dental aesthetics and the improvement in the masticatory function. There is a continuously increasing number of adult patients who actively seek orthodontic treatment, and it is also an undeniable fact that the incidence of periodontal disease increases with age. Therefore, the number of patients with periodontal problems that attend orthodontic practices is significantly greater than in the past. ${ }^{1}$

Orthodontic treatment can serve as an adjunct to periodontal treatment procedures to improve oral health in a number of situations. Pathological tooth migration is one of the few evident signs of periodontitis that affects dentofacial esthetics. This phenomenon is more commonly seen in the anterior dentition due to lack of stable occlusal and sagittal contacts with the opposing teeth., ${ }^{2,3}$

Achieving an esthetically acceptable result in such cases may require various orthodontic tooth movements like intrusion, rotation, and uprighting. This can also help control periodontal breakdown and restore good oral function. ${ }^{4}$

Fixed appliance therapy is more preferable if orthodontic tooth movement is desired in a patient suffering from periodontitis. Fixed appliance allows easy splinting of teeth to achieve stable anchorage, also it is important to reduce the force magnitude to reduce the stress on periodontal ligament fibres. ${ }^{5}$

Monitoring the levels of certain biochemical mediators may be a clinically useful procedure, to identify the degree of remodeling occurring in the periodontal tissues during orthodontic or orthopedic retention, because of their important roles in tooth movement and even in tissue damage. ${ }^{6}$

These biomarkers change in special clinical situations, such as severe periodontal disease, osseointegration, trauma due to orthodontic movements and are therefore indicative both of active pathology or healing. ${ }^{6}$
Gengival crevicular fluid (GCF) is an important source of biomarkers related to the state of health of deeper-seated tissues of periodontium. These properties can be particularly useful in monitoring the effectiveness of orthodontic treatment and the response of the alveolar bone to orthodontic forces. ${ }^{6}$

The importance of evaluating the levels of substances as valid biomarkers of periodontal effects of an orthodontic treatment is emphasized, through an accurate description of the specific role of each of them. ${ }^{6}$ Proinflammatory Cytokines: Interleukin$1 \beta$ (IL-1 $\beta)$, Interleukin-6 (IL-6), Interleukin-8 (IL-8), Tumor Necrosis Factor- $\alpha$ (TNF- $\alpha$ ), and Prostaglandin E (PGE). ${ }^{7}$

Interleukin-1ß (IL-1ß) is one of the most potent cytokines in periodontal environment during the initial stage of orthodontic tooth movement. The potential sources of IL-1ß during tooth movement include cells such as fibroblasts, macrophages, cementoblasts, cementoclasts, osteoblasts, and osteoclasts. ${ }^{8} \mathrm{IL}-1 \beta$ is secreted by osteoclasts as an immediate response to mechanical stress during the initial stage of orthodontic treatment, this Interleukin also determines the amount of tooth movement dependently on the efficiency of alveolar bone remodeling process. ${ }^{9}$

Moreover, IL- $1 \beta$ is one of the mediators of inflammation which induces the secretion of substances causing pain. Besides, IL-1B is produced by the periodontal ligament in a quantity sufficient to diffuse into the gingival crevicular fluid and has been identified as a biomarker of orthodontic movement. ${ }^{7}$ IL-1ß is also considered to be a powerful inducer of Interleukin-6 (IL-6) production; it overlaps with IL- 6 and Tumor Necrosis Factor- $\alpha$ (TNF- $\alpha$ ) in their actions. ${ }^{10}$ IL- 6 has an increased amount after $24 \mathrm{hrs} .{ }^{11}$ It regulates immune responses in inflammation sites. ${ }^{12}$ The levels of Interleukin- 8 (IL-8) increased at PDL tension sites and proposed it to be a triggering factor for bone remodeling. ${ }^{13} \mathrm{In}$ human gingival crevicular fluid many findings have confirmed increased levels of these proinflammatory 
cytokines involving in parodontal remodeling during orthodontic tooth movement. ${ }^{14,15}$

A study identified the role of prostaglandins E (PGE1 and PGE2) in stimulating bone resorption. ${ }^{16}$ The primary response to the pressure stimulus is the liberation of prostaglandins, an important chemicalmessenger. PGE2, specially, is able to mediate inflammatory responses and induces bone resorption by activating osteoclastic cells. ${ }^{17}$ prostaglandins directly stimulate osteoclast production and induce bone resorption. In addition, the GCF level of PGE2 reflect the biologic activity in periodontium during orthodontic tooth movement, and it is significantly higher in both tension and compression sides. ${ }^{18}$

The effects of orthodontic forces on teeth affected by periodontal disease have not been extensively studied. ${ }^{19}$ However, studies ${ }^{17,18}$ indicated that periodontally compromised teeth can withstand orthodontic forces without additional damage to the periodontium.

Since the levels of some biological markers seen to be associated with an increased risk of progression of periodontal disease and with orthodontic tooth movement, the aim of the present study was to evaluate the levels of IL1B,IL6,IL8 and prostaglandin in the GCF in patients with periodontal disease during early stage of orthodontic tooth movement.

\section{SUBJECTS AND METHODS}

The study included nine subjects with periodontally affected teeth submitted to orthodontic treatment at the orthodontic department faculty of dentistry, Al-azhar University, Girls branch. With age range $23-45$ years. The subjects were required to have their periodontal condition under control with radiographic evidence of bone loss. Subjects had to be in good general health and were excluded if they had any systemic condition that would influence the course of periodontal disease or treatment. The patient were not allowed to use anti-inflammatory drugs 6-months before the start of treatment. The study was approved by the ethical committee of the faculty, and all subjects signed an informed consent form prior to enrollment in the study.

Fixed preadjusted edgewise appliance Roth type with 0.022 inch slots were inserted, nickel titanium light archwires sizes that ranged between $0.012,0.014,0.016$ and 0.018 inch were used for levelling and alignment.

Gingival crevicular fluid (GCF) samples were collected from the healthy and periodontally affected teeth with paper points at base line, 3 days, 1,2,3,4,6 weeks from starting of orthodontic treatment.

The samples were collected during the morning, Supragingival plaque was carefully removed before sampling, sampled sites were isolated with self retainng retractors, suction tips and cotton rolls, and dried gently with an air syringe. GCF samples were collected using paper strips which were inserted 1 to $2 \mathrm{~mm}$ into the gingival crevice for 1-2 minutes, each site was sampled 2-3 times at 1 minute interval, and the samples were placed in Eppendorf tubes. The samples stored at $-20^{\circ} \mathrm{C}$ until analysis ${ }^{20}$.

\section{RESULTS}

Interleukin 6: In healthy teeth, the mean value gradually increased after 3 days and 1 week, then gradually decreased after 2 and 3 weeks. This was followed by an increase at 4 weeks, to levels higher than the baseline. The overall percent change (increase) was $34.68 \pm 11.71$.

In periodontally affected teeth, the mean value gradually increased after 3 days and reached its peak at 1 week, then gradually decreased in the following observation times to a level lower than baseline. The overall percent change (decrease) was $-11.98 \pm 3.78$.

Comparing healthy and periodontally affected teeth revealed a significant difference except at 4 weeks (Table1, Fig.1) 
TABLE (1) Interleukin 6 levels in healthy and periodontally affected teeth and significance of the difference between both groups using $t$ test

\begin{tabular}{|c|c|c|c|c|c|c|c|c|}
\hline \multicolumn{2}{|c|}{} & Base line & 3-days & 1 week & 2 week & 3 week & 4 week & Percent change \\
\hline \multirow{2}{*}{\begin{tabular}{c} 
Healthy teeth \\
\cline { 2 - 10 }
\end{tabular}} & Mean & 8.91 & 11.47 & 14.36 & 4.37 & 1.91 & 12.02 & 34.681 \\
\hline \multirow{2}{*}{$\begin{array}{c}\text { Periodontally } \\
\text { affected teeth }\end{array}$} & Mean & 13.55 & 19.74 & 21.79 & 14.83 & 13.92 & 11.87 & -11.98 \\
\cline { 2 - 10 } & SD & 1.53 & 1.41 & 3.74 & 0.93 & 1.04 & 2.02 & 3.78 \\
\hline \multicolumn{2}{c}{$t$ value } & 9.27 & 3.16 & 3.56 & 8.10 & 23.6 & 0.23 & 21.81 \\
\hline \multicolumn{2}{|c|}{ P value } & $<0.0001 *$ & $0.0067 *$ & $0.0061 *$ & $<0.0001 *$ & $<0.0001 *$ & $0.65^{\mathrm{ns}}$ & $<0.0001^{*}$ \\
\hline
\end{tabular}

Significance level $p<0.05$, * statistically significant, $n$ s=non-significant

Interleukin 8: In healthy teeth, the mean value gradually increased after 3 days and 1 week, then gradually decreased after 2 and 3 weeks. This was followed by an increase at 4 weeks, to levels still lower than baseline. The overall percent change (decrease) was $-10.7 \pm 2.8$.

In periodontally affected teeth, the mean value gradually increased after 3 days and 1 week, then gradually decreased after 2 and 3 weeks, then increased at 4 weeks to a level still lower than baseline. The overall percent change (decrease) was $-51.74 \pm 14.79$.

Comparing healthy and periodontally affected teeth revealed a significant difference except at 4 weeks (Table2, Fig.1)

TABLE (2) Interleukin 8 levels in healthy and periodontally affected teeth and significance of the difference between both groups using t test

\begin{tabular}{|c|c|c|c|c|c|c|c|c|}
\hline \multicolumn{2}{|c|}{} & Base line & 3-days & 1 week & 2 week & 3 week & 4 week & Percent change \\
\hline \multirow{2}{*}{ Healthy teeth } & Mean & 1273.7 & 1839.2 & 2081.2 & 897.0 & 742.3 & 1146.6 & -10.7 \\
\cline { 2 - 10 } & SD & 26.2 & 51.7 & 72.8 & 37.6 & 47.2 & 204.6 & 2.8 \\
\hline \multirow{2}{*}{$\begin{array}{c}\text { Periodontally } \\
\text { affected teeth }\end{array}$} & Mean & 2225.7 & 2553.5 & 2782.6 & 2142.8 & 944.8 & 1074.2 & -51.74 \\
\cline { 2 - 10 } & SD & 82.6 & 234.9 & 138.9 & 54.8 & 70.8 & 49.1 & 1.26 \\
\hline \multirow{2}{*}{$t$ value } & 34.74 & 36.21 & 26.87 & 59.27 & 7.526 & 1.088 & 35.65 \\
\hline \multicolumn{2}{c}{ P value } & $<0.0001^{*}$ & $<0.0001^{*}$ & $<0.0001^{*}$ & $<0.0001^{*}$ & $<0.0001^{*}$ & $0.291^{\mathrm{ns}}$ & $<0.0001^{*}$ \\
\hline
\end{tabular}

Significance level p<0.05, * statistically significant, $n$ s=non-significant 
Interleukin 1B: In healthy teeth, the mean value gradually increased after 3 days and 1 week, then gradually decreased after 2 and 3 weeks. This was followed by an increase at 4 weeks, to levels higher than baseline. The overall percent change (increase) was $80.05 \pm 12.01$.

In periodontally affected teeth, the mean value gradually increased after 3 days and 1 week, then gradually decreased after 3 and 4 weeks, to a level lower than baseline. The overall percent change (decrease) was $-54.51 \pm 4.43$.

Comparing healthy and periodontally affected teeth revealed a significant difference except at 1 week (Table3, Fig.1)
Prostaglandin: In healthy teeth, the mean value gradually increased after 3 days and 1 week, then gradually decreased after 2, 3 and 4weeks to a level higher lower than baseline. The overall percent change (decrease) was $-9.46 \pm 3.12$.

In periodontally affected teeth, the mean value increased after 3 days, and reached its peak at 1 week, then gradually decreased after 2 weeks and the subsequent observation times, to a level lower than baseline at 4 weeks. The overall percent change (increase) was $-17.07 \pm 4.33$.

Comparing healthy and periodontally affected teeth revealed a significant difference at all intervals (Table4, Fig.1)

TABLE (3) Interleukin 1B levels in healthy and periodontally affected teeth and significance of the difference between both groups using $t$ test

\begin{tabular}{|c|c|c|c|c|c|c|c|c|}
\hline \multicolumn{2}{|c|}{} & Base line & 3-days & 1 week & 2 week & 3 week & 4 week & percent change \\
\hline \multirow{2}{*}{\begin{tabular}{l} 
Healthy teeth \\
\cline { 2 - 10 }
\end{tabular}} & Mean & 8.12 & 17.03 & 21.75 & 8.35 & 5.75 & 14.62 & 80.05 \\
\hline \multirow{2}{*}{$\begin{array}{c}\text { Periodontally } \\
\text { affected teeth }\end{array}$} & Mean & 17.47 & 19.32 & 23.97 & 23.70 & 11.62 & 7.93 & -54.51 \\
\cline { 2 - 10 } & SD & 0.40 & 2.18 & 3.20 & 2.75 & 1.21 & 0.61 & 4.43 \\
\hline \multicolumn{2}{|c|}{$t$ value } & 56.32 & 2.148 & 1.924 & 17.49 & 13.78 & 15.41 & 19.06 \\
\hline \multicolumn{2}{|c|}{ P value } & $<0.0001 *$ & $0.0455^{*}$ & $0.07^{\mathrm{ns}}$ & $<0.0001 *$ & $<0.0001 *$ & $<0.0001 *$ & $<0.0001 *$ \\
\hline
\end{tabular}

Significance level $p<0.05$, statistically significant, $n s=n o n-$ significant

TABLE (4) Prostaglandin levels in healthy and periodontally affected teeth and significance of the difference between both groups using $\mathrm{t}$ test

\begin{tabular}{|c|c|c|c|c|c|c|c|c|}
\hline \multicolumn{2}{|c|}{} & Base line & 3-days & 1 week & 2 week & 3 week & 4 week & Percent change \\
\hline \multirow{2}{*}{\begin{tabular}{c} 
Healthy teeth \\
\cline { 2 - 10 }
\end{tabular}} & Mean & 54.12 & 59.61 & 70.31 & 62.07 & 51.05 & 49.00 & -9.46 \\
\hline \multirow{2}{*}{$\begin{array}{l}\text { Periodontally } \\
\text { affected teeth }\end{array}$} & Mean & 73.47 & 84.33 & 87.67 & 71.48 & 70.59 & 60.93 & -17.07 \\
\cline { 2 - 11 } & SD & 5.92 & 4.40 & 3.93 & 7.25 & 5.92 & 6.44 & 4.33 \\
\hline \multicolumn{2}{|c|}{$t$ value } & 6.99 & 8.21 & 5.94 & 6.43 & 17.83 & 3.872 & 3.96 \\
\hline \multicolumn{2}{|c|}{ P value } & $<0.0001 *$ & $<0.0001 *$ & $<0.0001 *$ & $<0.0001 *$ & $<0.0001 *$ & $<0.0001 *$ & $0.002 *$ \\
\hline
\end{tabular}

Significance level $p<0.05$, * statistically significant 


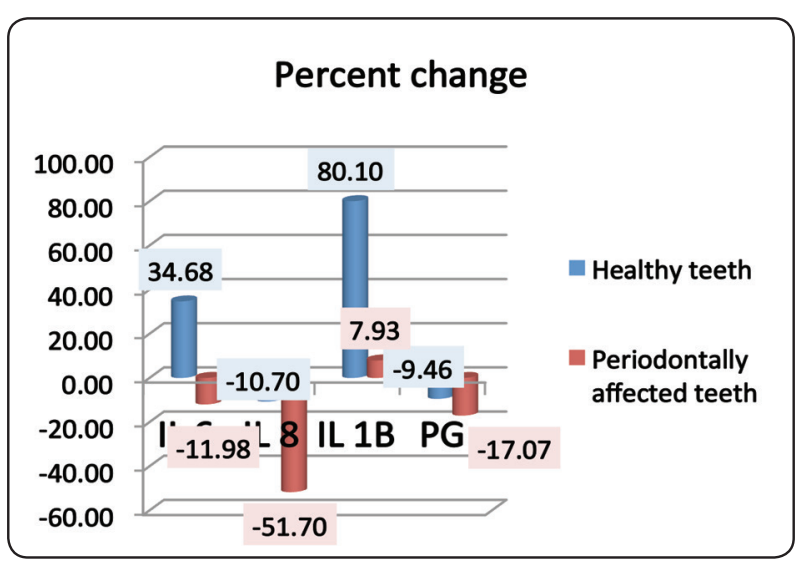

Fig. (1) Column chart showing mean percent change in biomarker levels in healthy and periodontally affected teeth throughout the study

\section{DISCUSSION}

Alveolar bone and adjacent periodontal tissues show extensive macroscopic and microscopic changes when exposed to varying degrees of magnitude, frequency, and duration of mechanical loading. Mechanical loading also alters periodontal tissue vascularity and blood flow, resulting in the local synthesis and release of various molecules, such as cytokines, growth factors, colony-stimulating factors, enzymes, and neurotransmitters. ${ }^{7}$

On the basis of sequential reactions and released substances, several of these biologically active molecules have been proposed as biomarkers to better understand the biological process involved in orthodontic tooth movement, to improve treatment and reduce adverse side effects. ${ }^{21}$

A biomarker is a substance measured and evaluated objectively as an indicator of physiologic processes, pathogenic processes, or responses to a therapeutic treatment. ${ }^{22}$

The biological mechanisms controlling the shift from the stimulus, consisting of continuous force application, to the reaction, represented by the displacement of the tooth in the periodontal space, could be evaluated by monitoring the higher or lower rate of such biomarkers in the periodontium. ${ }^{7}$
Potential biological markers can be collected by means of different experimental and clinical methods, respectively, in animal and in living human models. GCF analysis, especially, has offered several advantages for its simple, quick, and noninvasive collection and for the wide variety of molecules detected in his volume. ${ }^{7}$

GCF is a powerful vehicle for clinical diagnostics, since it contains different biochemical and cellular arrays in relation to different clinical situations indicative of the state of periodontal health during orthodontic treatment. ${ }^{6}$

In this study, Days 7 and 21 were chosen for GCF sampling because day 7 is the turnover time for enzymes and indirect resorption processes start on day $21 .^{23}$

The main gingival crevicular fluid (GCF) biomarkers related to orthodontic tooth movements were classified into four main groups: biomarkers of inflammation, bone metabolism, cell death and bone deposition and mineralization. Biomarkers of inflammation are Interleukins (IL-1 $\beta$, IL-6, IL-8), Tumour Necrosis factors (TNF- $\alpha$ ), Colony-stimulating factors (M-CSF, G-CSF, GM-CSF), Prostaglandins (PGE), Vascular endothelial growth factors (VEGF), Calcitonin gene related peptide (CGRP), Substance P. Interleukins (IL-1 $\beta$, IL-6, IL-8) are cytokines involved in the bone remodelling, bone resorption and neo-bone apposition during orthodontic tooth movement. ${ }^{7,24,25}$

These biomarkers change in special clinical situations, such as severe periodontal disease, osseointegration, trauma due to orthodontic movements and are therefore indicative both of active pathology or healing. ${ }^{6}$

Our results showed that Interleukin 1 in healthy teeth, the mean value gradually increased after 3 days and 1 week, then gradually decreased after 2 and 3 weeks. This was followed by an increase at 4 weeks, to levels higher than baseline. In periodontally 
affected teeth, the mean value gradually increased after 3 days and 1 week, then gradually decreased after 3 and 4 weeks, to a level lower than baseline. Comparing healthy and periodontally affected teeth revealed a significant difference except at 4 weeks, this is in agreement with a study revealed that, there was a significant higher level of IL- $1 \beta$ in the diseased sites than the non diseased sites. ${ }^{26}$ The IL- $1 \beta$ is initially released to compat infection, its expression is influenced by the microbial composition of the adjacent gingival biofilm. These results suggest that IL- $1 \beta$ is currently the most useful biomarker. ${ }^{26} \mathrm{~A}$ study reavelrd that IL- $1 \beta$ is secreted by osteoclasts as an immediate response to mechanical stress during the initial stage of orthodontic treatment, and at later stages by macrophages, whose accumulation has been observed in compressed areas. This Interleukin also determines the amount of tooth movement dependently on the efficiency of alveolar bone remodeling process, ${ }^{27}$ IL- $1 \beta$ is produced by the periodontal ligament in a quantity sufficient to diffuse into the gingival crevicular fluid and has been identified as a biomarker of orthodontic movement. ${ }^{6}$ A review article identify the bone resorption marker, ${ }^{21}$ The earliest bone resorption marker is the interleukin- 1 beta (IL-1 $\beta$ ), PGE2, interleukin-6 (IL-6), and other inflammatory cytokines can also facilitate osteoclastic bone resorption processes.$^{28}$

Interleukin-6 (IL-6) is a multifunctional cytokine, and increased levels are involved in bone destruction. ${ }^{25}$ Interleukin-6 in this study showed gradual increase In healthy teeth after 3 days and 1 week, then gradually decreased after 2 and 3 weeks. This was followed by an increase at 4 weeks, In periodontally affected teeth, the mean value gradually increased after 3 days and reached its peak at 1 week, then gradually decreased in the following observation times to a level lower than baseline. Regarding healthy and periodontally affected teeth there are significant higher level, this is in agreement with findings of studies reported increased level during orthodontic tooth movement from the first hour and decreased after 7 and 10 days..$^{8,25,29}$
Interleukin-8 plays a multifunctional roles in the pathogenesis of periodontal disease, it plays a major role in mediating inflammatory responces, orthodontic forces evoke changes in the level of Interleukin- $8{ }^{13}$ In this study the level of Interleukin-8 in healthy teeth, gradually increased after 3 days and 1 week, then gradually decreased after 2 and 3 weeks. This was followed by an increase at 4 weeks, to levels still lower than baseline. In periodontally affected teeth, the mean value gradually increased after 3 days and 1 week, then gradually decreased after 2 and 3 weeks, then increased at 4 weeks to a level still lower than baseline. Comparing healthy and periodontally affected teeth revealed a significant difference except at 4 weeks. This is in agreement with studies ${ }^{13,23,25}$ reported significant increase in Interleukin-8 during orthodontic tooth movement beginning from first hour and reached maximum level at day 6 and another study reported an increase at day 7 and 21 during canine retraction.

Prostaglandin PG, has long been considered a major stimulator of bone resorption, ${ }^{30}$ it is induced by interleukin- $1 \beta$. IL- $1 \beta$ synergically upregulate the formation of prostaglandins in the periodontal tissue subjected to orthodontic stress. Orthodontic and orthopaedic forces evoke changes in the levels of inflammatory mediators in the periodontal tissues and can trigger the processes of bone resorption in tissue around the teeth. ${ }^{31}$ A study reported an increased levels of PGE adjacent to teeth undergoing orthodontic movement with fixed appliances, 32 which is compatible with our finding that showed gradual increase of PG at 3 days and reaches its peak after one week of orthodontic treatment. In periodontally affected teeth the PG showed higher level than in non affected teeth, indicating more inflammation and bone resorption. prostaglandins directly stimulate osteoclast production and their capacity to form ruffled border and effect bone resorption. In addition, the GCF level of PGE2 reflect the biologic activity in periodontium during orthodontic tooth movement, and it is significantly higher in both tension and compression sides. ${ }^{33}$ 
Conclusion: gingival crevicular fluids are common, non-invasive methods of collecting samples associated with orthodontic tooth movement. Gingival crevicular fluid (GCF) can reflect the state of health of the periodontal tissues. The biomarkers level increased with orthodontic tooth movement in the first week then gradually decreased in both healthy and periodontally affected teeth, the level is higher in periodontally affected than healthy teeth.

The clinical use of biomarkers in orthodontics is still an issue. Biomarkers remain a challenging task specially in case of orthodontic treatment of periodontally affected teeth.

\section{CONFLICT OF INTERESTS}

The authors declare that there is no conflict of interests regarding the publication of this paper

\section{REFERENCES}

1. Gkantidis N, Christou P, Topouzelis N. The orthodonticperiodontic interrelationship in integrated treatment challenges: a systematic review. J Oral Rehabilitation 2010;37:377-390.

2. Gomes SC, Varela CC, da Veiga SL, Rösing CK, Oppermann RV. Periodontal conditions in subjects following orthodontic therapy. A preliminary study. Eur J Orthod. 2007;29:477-81.

3. Vinod K, Reddy YG, Reddy VP, Nandan H, Sharma M. Orthodontic-periodontics interdisciplinary approach. J Indian Soc Periodontol. 2012;16:11-15.

4. Zachrisson BU. Orthodontics and periodontics. In: Lindhe J, Karring T, Lang NP. Clinical periodontology and implant dentistry. 4th ed. Oxford: Blackwell Munksgaard; 2003:744-80.

5. Proffit WR, Sarver DM. Special considerations in treatment for adults. In: Proffit WR, Fields HW, Sarver DM. Contemporary orthodontics. $5^{\text {th }}$ ed. St. Louis: Mosby; 2013:623-661.

6. Lauritano D., Avantaggiato A, Cura F, GirardiA, Carinci F. Biomarkers of periodontal tissue in gingival crevicular fluid during orthodontic movements: An overview. OA Dentistry 2014; Jan 18;2(1):1-4.

7. Apuzzo F, Cappabianca S, and omenico Ciavarella D, Monsurrò A, Silvestrini-Biavati A, and Perillo L.
Biomarkers of Periodontal Tissue Remodeling during Orthodontic Tooth Movement in Mice and Men: Overview and Clinical Relevance. ScientificWorld J, 2013, Article ID 105873,8 pages

8. Alhashimi N, Frithiof L, Brudvik P, and Bakhiet M. Orthodontic tooth movement and de novo synthesis of proinflammatory cytokines. Am J Orthod and Dentofacial Orthop. 2001;119: 307-12.

9. Teixeira CC, Khoo E, Tran J et al. Cytokine expression and accelerated tooth movement. J Dental Research, 2010; 89:1135- 41 .

10. Uematsu, Mogi SM, and Deguchi T. Interleukin (IL)$1 \beta$, IL- 6 , tumor necrosis factor- $\alpha$, epidermal growth factor, and $\beta 2$ - microglobulin levels are elevated in gingival crevicular fluid during human orthodontic tooth movement. J Dental Research. 1996;75:562-567.

11. Linkhart TA, Linkhart SG, MacCharles DC, Long DL, and Strong D. D. Interluekin-6 messenger RNA expression and interleukin- 6 protein secretion in cells isolated from normal human bone: regulation by interleukin-1. J Bone and Mineral Research. 1991;6:1285-94.

12. Okada N, Kobayashi M, Mugikura K, Okamatsu Y, Hanazawa S, KitanoS, Hasegawa K. Interleukin-6 production in human fibroblasts derived from periodontal tissues is differentially regulated by cytokines and a glucocorticoid. J Periodontal Research. 1997;32:559-569.

13. Tuncer B, Ozmeric N, Tuncer C. et al. Levels of interleukin-8 during tooth movement. Angle Orthod. 2005; 75: 539-544.

14. 14. Lowney JJ, Norton LA, Shafer DM, Rossomando EF. Orthodontic forces increase tumor necrosis factor $\alpha$ in the human gingival sulcus. Am J Orthod Dentofacial Orthop 1995;108:519-24.

15. Grieve WG, Johnson GK, Moore RN, Reinhardt RA, DuBois LM. Prostaglandin E (PGE) and interleukin-1 beta (IL-1 beta) levels in gingival crevicular fluid during human orthodontic tooth movement. Am J Orthod and Dentofac Orthop.1994;105:369-74.

16. Lee W. Experimental study of the effect of prostaglandin administration on tooth movement-with particularemphasis on the relationship to the method of PGE1 administration. Am J Orthod Dentofac Orthop. 1990;98:238-41.

17. Krishnan V. and Davidovitch Z. Cellular, molecular, and tissue-level reactions to orthodontic force. Am J Orthod and Dentofac Orthop. 2006;129;469-e1-469-e32. 
18. Dudic A, Kiliaridis S, Mombelli A. and Giannopoulou C. Composition changes in gingival crevicular fluid during orthodontic tooth movement: comparisons between tension and compression sides. Eur J of Oral Sciences. 2006; 114:416-422.

19. 19. Almeidaa RC. Capelli JJ, Telesb RP. Levels of gingival crevicular fluid matrix metalloproteinases in periodontally compromised teeth under orthodontic forces. Angle Orthod 2015;85:1009-1014.

20. Yamamoto Z., Jaafar I.M., Wahab Z.Z., Abidin I.Z.Z., Senafi S, Ariffin S.H.Z. profiles of inflammatory markers for traumatized and non traumatized incisors during orthodontic treatment with two archwire changes. J Biological sciences 2014;14:299-304.

21. Ariffin SHZ, Yamamoto Z, Abidin IZZ, AbdulWahab RM, AriffinZ Z. Cellular and molecular changes in orthodontic tooth movement. Scientific World J. 2011;11:1788-1803.

22. Taba M, Kinney J, Kim AS, Giannobile WV. Diagnostic biomarkers for oral and periodontal diseases. Dental Clinics of North America. 2005;49:551-71.

23. Basaran G, Ozer T, Kaya FA, Kaplan A, Hamamci O. Interleukin-1 beta and tumor necrosis factor-alpha levels in the human gingival sulcus during orthodontic treatment. Angle Orthod. 2006;76:830-36.

24. Kunii R, Yamaguchi M, Tanimoto Y, Asano M, Yamada K, Goseki T, Kasai K. Role of interleukin-6 in orthodontically induced inflammatory root resorption in humans. Korean J Orthod. 2013, 43:294.

25. Hamamci N, Acun Kaya F, Uysal E, Yokus B. Identification of interleukin 2, 6, and 8 levels around miniscrews during orthodontic tooth movement. Eur J Orthod. 2012, 34:357-361.
26. Shimada Y, Tabeta K, Sugita N, Yoshie H. profiling biomarkers in gingival crevicular fluid using multiplex bead immunoassay. Archives of oral biology. 2013;58:724-30.

27. Teixeira CC, Khoo E, Tran J. et al. Cytokine expression and accelerated tooth movement. J Dental Research. 2010;89: 1135-41.

28. Masella RS, Meister M. Current concepts in the biology of orthodontic tooth movement. Am J Orthod Dentofacial Orthop. 2006;129:458-68.

29. Davidovitch Z, Nicolay O, Ngan PW and Shanfield JL. Neurotransmitters, cytokines and the control of alveolar bone remodeling in orthodontics. Dental clinics of North America. 1988;32:411-35.

30. Mathew S. orthodontic tooth movement and changes in gingival crevicular fluid: A Review. J Ind Orthod Soc 2004;37:102-14.

31. Chibebe PC, Starobinas N, Pallos D. Juveniles versus adults: differences in PGE2 levels in the gingival crevicular fluid during orthodontic tooth movement. Braz Oral Res 2010, 24:108-13.

32. Grieve WG, Johnson GK, Moore RN, Reinhardt RA, DuBois EM. Prostaglandin E (PGE) and Interleukins1a(IL-1a) levels in gingival crevicular fluid during human orthodontic tooth movement. Am J Orthod Dentofacial Orthop 1994;105:368-74.

33. Dudic A, Kiliaridis S, Mombelli A, Giannopoulou C. Composition changes in gingival crevicular fluid during orthodontic tooth movement: comparisons between tension and compression sides. Eur $\mathbf{J}$ of Oral Sciences 2006;114: 416-422. 